\title{
SYMBOLIC DYNAMICS AND TRANSFORMATIONS OF THE UNIT INTERVAL
}

\author{
BY \\ WILLIAM PARRY
}

0. Introduction. This paper extends some results from [1] and applies them to certain transformations of the unit interval. $\S \S 2-4$ are concerned with symbolic dynamics and $\$ \S 5-6$ are concerned with their application to the proof of our main theorem which states sufficient conditions for a piecewise continuous transformation of the unit interval to be conjugate to a (uniformly) piecewise linear transformation.

The main result includes a classical theorem of Poincaré-Denjoy [2] on homeomorphisms of the circle onto itself. It also provides a partial answer to a question of Ulam's [3] concerning the possibility of piecewise linearising continuous transformations of the unit interval. This problem was also mentioned by Stein and Ulam in [4], together with the remark that necessary conditions can be given in terms of the trees of points, but that no meaningful sufficient conditions are known. In the same work a few special examples are examined. Our main theorem also has a bearing on certain transformations discussed by Rényi [5].

In $\S \S 2-4$ we consider the shift transformation acting on a compact invariant subset of the space of one-way infinite sequences of symbols chosen from a finite set. The shift transformation on such a set is continuous but not necessarily open. If $X, T$ are the compact invariant set and the shift transformation, respectively, we refer to $(X, T)$ as a symbolic dynamical system [6]. For a symbolic dynamical system $(X, T)$ we define a number called the absolute entropy $\left({ }^{1}\right)$ which dominates the entropy of $T$ with respect to each normalised $T$ invariant Borel measure, and show that if $T$ is regionally transitive then there is always one invariant measure with respect to which the entropy of $T$ equals the absolute entropy of $T$. When $T$ is open, (or equivalently, when $(X, T)$ is an intrinsic Markov chain) this "maximal" measure is unique. A further theorem states that, under certain conditions, there exists a normalised Borel measure with respect to which $T$ acts in a "linear" fashion.

In $§ \S 5-6$ we apply this latter theorem to certain transformations of the unit interval and obtain our main result, Theorem 5 .

Received by the editors October 5, 1965 .

(1) For such systems the absolute entropy and the topological entropy of Adler,Konheim and McAndrew [7] can be shown to coincide. 
1. Definitions. Let $X_{0}=\prod_{n=0}^{\infty} Z_{n}$ where $Z_{n}=Z=(0,1, \cdots, s) \quad(s \geqq 1)$. We consider $X_{0}$ as a topological space endowed with the compact metric totally disconnected product topology which arises from the discrete topology on $Z$. Let $T_{0}$ denote the shift transformation $T_{0} x=x^{\prime}$ where $x=\left\{Z_{n}(x)\right\}, x^{\prime}=\left\{Z_{n}\left(x^{\prime}\right)\right\}$, $Z_{n}\left(x^{\prime}\right)=Z_{n+1}(x)$. By a symbolic dynamical system we mean a pair $(X, T)$ where $X$ is a compact $T_{0}$ invariant $\left(T_{0} X=X\right)$ subset of $X_{0}$ and $T$ is the restriction of $T_{0}$ to $X$. We shall be interested in normalised Borel measures defined on the Borel field generated by cylinders of $X$. A cylinder of $X_{0}$ is a set of the form

$$
C \equiv\left\{x: Z_{i}(x)=a_{i}, 0 \leqq i \leqq n\right\} \equiv\left(a_{0}, \cdots, a_{n}\right) .
$$

A cylinder of $X$ is a set of the form $C \cap X$ where $C$ is a cylinder of $X_{0}$. We assume, as we may, that the cylinders $(i) \cap X$ are not empty for $i=0,1, \cdots, s$. An important property of the relative topology of $X$ is that cylinders are both open and closed. $T$ is continuous but not necessarily open.

We shall always impose the following condition of regional transitivity on $(X, T)$ :

(1.1) For every pair of nonempty cylinders $C, D$ of $X$ there exists an integer $n$ such that

$$
T^{n} C \cap D \neq \varnothing \text { or equivalently } C \cap T^{-n} D \neq \varnothing .
$$

Condition (1.1) is satisfied if:

(1.2) For every nonempty cylinder $C$ of $X$

$$
\bigcup_{n=0}^{\infty} T^{n} C=X .
$$

One can prove that (1.1) and (1.2) are equivalent when $T$ is open.

Let $X^{\prime}$ be the unit interval with or without each end point. Let

$$
0=a_{0}<a_{1}<\cdots<a_{s+1}=1
$$

$(s \geqq 1)$ and $A^{\prime}(i)=\left(a_{i}, a_{i+1}\right)$ and let $T^{\prime}$ be a transformation of $X^{\prime}$ onto itself such that for each $i=0,1, \cdots, s, T^{\prime}$ is either strictly increasing and continuous on $A^{\prime}(i)$ or strictly decreasing and continuous on $A^{\prime}(\mathrm{i})$. Suppose also that $T$ is continuous from the right at 0 if $0 \in X^{\prime}$, continuous from the left at 1 if $1 \in X^{\prime}$ and at each $a_{i}, T^{\prime}$ is either continuous from the left or continuous from the right. $T^{\prime}$ is then called a piecewise monotonic transformation of $X^{\prime}$ onto itself.

A piecewise monotonic transformation $T^{\prime}$ is called uniformly piecewise linear if there exists $\beta \geqq 1,\left\{\alpha_{i}\right\}$ such that

$$
T^{\prime}(x)=\alpha_{i} \pm \beta x^{\prime} \quad \text { for } \quad x^{\prime} \in A^{\prime}(i)
$$

and the sign involved is constant for each $i=0,1, \cdots, s$.

A transformation $T^{\prime}$ is called strongly transitive if for every nonempty open set $U$ there exists an integer $m$ such that 


$$
\bigcup_{n=0}^{m} T^{\prime n} U=X^{\prime}
$$

Two transformations $S^{\prime}, T^{\prime}$ of $X^{\prime}$ onto itself are said to be conjugate if there exists a homeomorphism $\phi$ of $X^{\prime}$ onto itself such that

$$
S^{\prime}=\phi T^{\prime} \phi^{-1}
$$

2. Intrinsic Markov chains. A symbolic dynamical system $(X, T)$ is said to be an intrinsic Markov chain of order $r$ if the following condition is satisfied:

(2.1) If $\left(a_{0}, \cdots, a_{n}\right) \cap X \neq \varnothing(n \geqq r)$ and $\left(a_{n-r+1} \cdots a_{n} a_{n+1}\right) \cap X \neq \varnothing$ implics $\left(a_{0} \cdots a_{n-r+1} \cdots a_{n} a_{n+1}\right) \cap X \neq \varnothing$.

Before stating our result concerning intrinsic Markov chains we prove the following.

THeOrem 1. A symbolic dynamical system $(X, T)$ is an intrinsic Markov chain if and only if $T$ is open.

Proof. Suppose $(X, T)$ is an intrinsic Markov chain of order $r$ and let $\left(a_{0}, \cdots, a_{n}\right) \cap X \neq \varnothing$ where $n \geqq r$. It will suffice to show that $T\left[\left(a_{0} \cdots a_{n}\right) \cap X\right]$ $=\left(a_{1} \cdots a_{n}\right) \cap X$. Obviously $T\left[\left(a_{0} \cdots a_{n}\right) \cap X\right] \subset\left(a_{1} \cdots a_{n}\right) \cap X$. Suppose $x \in\left(a_{1} \cdots a_{n}\right) \cap X$; we have to show that there exists a point $y \in\left(a_{0} \cdots a_{n}\right) \cap X$ with $T y=x$, or in other words, that $a_{0} \cdots a_{n} \cdots \in X$ if $x=a_{1} a_{2} \cdots a_{n} \cdots$. But $\left(a_{0} \cdots a_{n}\right) \cap X \neq \varnothing$ and $x \in\left(a_{1} \cdots a_{n+1}\right) \cap X \neq \varnothing$. Consequently $T^{n-r} x \in\left(a_{n-r+1} \cdots a_{n+1}\right) \cap X \neq \varnothing$ and since $(X, T)$ is an intrinsic Markov chain of order $r$ we have $\left(a_{0} \cdots a_{n+1}\right) \cap X \neq \varnothing$. Repeating this argument indefinitely we have, for every $k,\left(a_{0} \cdots a_{k}\right) \cap X \neq \varnothing$ and the point $y=a_{0} \cdots a_{n} a_{n+1} \cdots \in X$ where $x=a_{1}, a_{2} \cdots$.

Suppose the shift transformation $T$ of $X$ onto itself is open. We first show that every point $x=a_{0} a_{1} \cdots \in X$ belongs to a cylinder $\left(a_{0} \cdots a_{n}\right)$ such that;

$$
T\left[\left(a_{0} \cdots a_{n}\right) \cap X\right]=\left(a_{1} \cdots a_{n}\right) \cap X .
$$

Quite generally one can show that

$$
T\left[\left(a_{0} \cdots a_{n}\right) \cap X\right]=\left(a_{1} \cdots a_{n}\right) \cap T\left[\left(a_{0}\right) \cap X\right] .
$$

Obviously $T x \in T\left[\left(a_{0}\right) \cap X\right]$ and the latter is open. We have to show that $X \cap\left(a_{1} \cdots a_{n}\right) \subset T\left[\left(a_{0}\right) \cap X\right]$ for some $n$. If this were not true then the decreasing sequence of nonempty closed sets $X \cap\left(a_{1} \cdots a_{n}\right)-T\left[\left(a_{0}\right) \cap X\right]$ would have a nonempty intersection, and this is impossible since the only point in all the sets $X \cap\left(a_{1} \cdots a_{n}\right)$ is $T x$ and this point belongs to $T\left[\left(a_{0}\right) \cap X\right]$.

For each point $x \in X$ let $S(x)$ denote the nonempty cylinder $\left(a_{0} \cdots a_{n}\right) \cap X$ of smallest length which satisfies (2.2). Since $X$ is compact a finite number of the cynliders $S(x)$ will cover $X$. If $X \cap\left(a_{0} \cdots a_{n}\right) \neq \varnothing$ satisfies (2.2) and 
$X \cap\left(a_{0} \cdots a_{n+k}\right) \neq \varnothing$ then $X \cap\left(a_{0} \cdots a_{n+k}\right)$ satisfies (2.2). Therefore there exists a smallest integer $n$ such that $X$ can be partitioned into a set $\mathscr{P}$ of nonempty cylinders $X \cap\left(a_{0} \cdots a_{n}\right)$ of the same length $n$ each satisfying (2.2). It follows from this that for every $C \in \mathscr{P}, T C$ is a disjoint union of sets from $\mathscr{P}$. This property will ensure that $(X, T)$ is an intrinsic Markov chain of order $(n+1)$.

3. Maximal measures for intrinsic Markov chains. Let $\mu$ be a normalised invariant measure for a symbolic dynamical system $(X, T)$. The entropy of $T$ with respect to $\mu$ is defined as

$$
h_{\mu}(T)=\lim _{n \rightarrow \infty}-\frac{1}{n} \sum \mu(C) \log \mu(C)
$$

where the summation is over cylinders of length $n$. The absolute entropy of $T$ is defined as

$$
e(T)=\lim _{n \rightarrow \infty} \frac{1}{n} \log \theta(n)
$$

where $\theta(n)$ is the number of nonempty cylinders of $X$ of length $n$. It is easy to verify that for all normalised invariant measures $\mu$

$$
h_{\mu}(T) \leqq e(T) .
$$

THEOREM 2 [1]. If $(X, T)$ is a regionally transitive intrinsic Markov chain of order $r$ then there exists one and only one normalised Borel invariant measure $\mu$ such that $e(T)=h_{\mu}(T)$. With respect to $\mu,(X, T)$ is a multiple Markov chain of order $r$ and the regional transitivity ensures that $T$ is ergodic with respect to $\mu$. Moreover, there exists a normalised measure $p$ equivalent to $\mu$ and a number $\beta>1$ (in fact, $e(T)=\log \beta$ ) such that for all cylinders $C$ of $X, p T C=\beta p C$. The measures $p, \mu$ are nonatomic and are positive on nonempty cylinders of $X$.

We are interested in extending the above theorem to the case where $(X, T)$ is not intrinsically Markovian, i.e. to the case where $T$ is not open. The theorems which follow only partially solve this problem.

THEOREM 3. If $(X, T)$ is a regionally transitive symbolic dynamical system then there exists a normalised Borel invariant measure $\mu$ (with respect to which $T$ is ergodic) such that

$$
h_{\mu}(T)=e(T) .
$$

Proof. We omit the proof that $T$ is ergodic with respect to some maximal measure but remark that this can be shown by decomposing a maximal measure into its ergodic parts and using the affinity of $h_{\mu}(T)$ as a function of $\mu[8$, p. 183].

Let $X_{n}=\left\{x \in X_{0}:\left(Z_{k}(x) \cdots Z_{k+n}(x)\right) \cap X \neq \varnothing\right.$ for all $\left.k\right\}$. We consider the symbolic dynamical systems $\left(X_{n}, T_{n}\right)$, where $T_{n}$ is the restriction of $T$ to $X_{n}$. It is easily verified that the sets $X_{n}$ are compact and $T_{n}$ invariant. Moreover, by con- 
struction, $\left(X_{n}, T_{n}\right)$ is a regionally transitive intrinsic Markov chain of order $n$, $X_{n} \supset X_{n+1}$ and $\bigcap_{n} X_{n}=X$.

It is easy to show that $e\left(T_{n}\right) \geqq e\left(T_{n+1}\right) \geqq e(T)$, and in fact that $e\left(T_{n}\right) \rightarrow e(T)$.

A well-known formula [9] for $h_{\mu}\left(T_{n}\right)$ is

$$
h_{\mu}\left(T_{n}\right)=H\left(\mathscr{A}_{n} \mid \bigvee_{i=1}^{\infty} T_{n}{ }^{-i} \mathscr{A}_{n}\right)
$$

where $\mathscr{A}_{n}$ is the partition (0) $\cap X_{n},(1) \cap X_{n}, \cdots,(s) \cap X_{n}$.

Let $\mu$ be a limit point of the maximal measures $\mu_{n}$, defined by Theorem 2 for the systems $\left(X_{n}, T_{n}\right)$, on the dual space of the space of continuous functions on $X_{0}$. Then there exists a sequence of integers $m(n)$ such that for all continuous functions $f(x)$ defined on $X_{0}$

$$
\int f(x) d \mu_{m(n)} \rightarrow \int f(x) d \mu,
$$

and since characteristic functions of cylinders are continuous we have

$$
\mu_{m(n)}(C) \rightarrow \mu(C)
$$

for all cylinders $C$ of $X_{0}$. It is clear that $\mu$ is concentrated on $X$ since $\mu(C)=0$ for cylinders $C$ of $X_{0}$ such that $C \cap X=\varnothing$.

Choose $\varepsilon>0$ and $k=k(\varepsilon), n=n(k)$ so that

$$
\begin{aligned}
h_{\mu}(T) & \geqq H_{\mu}\left(\mathscr{A} \mid T^{-1} \mathscr{A} \vee \cdots \vee T^{-k} \mathscr{A}\right)-\varepsilon \\
& =H_{\mu}\left(\mathscr{A}_{0} \vee \cdots \vee T_{0}^{-k} \mathscr{A}_{0}\right)-H_{\mu}\left(\mathscr{A}_{0} \vee \cdots \vee T_{0}^{(1-k)} \mathscr{A}_{0}\right)-\varepsilon \\
& \geqq H_{\mu_{n}}\left(\mathscr{A}_{0} \vee \cdots \vee T_{0}^{-k} \mathscr{A}_{0}\right)-H_{\mu_{n}}\left(\mathscr{A}_{0} \vee \cdots \vee T_{0}^{(1-k)} \mathscr{A}_{0}\right)-2 \varepsilon
\end{aligned}
$$

Here we have used the continuity of $H$ as a function of normalised measures and the facts that $\mu_{n}, \mu$ are concentrated on $X_{n}, X$ respectively.

Hence

$$
\begin{aligned}
h_{\mu}(T) & \geqq H_{\mu_{n}}\left(\mathscr{A}_{0} \mid T_{0}^{-1} \mathscr{A}_{0} \vee \cdots \vee T^{-k} \mathscr{A}_{0}\right)-2 \varepsilon \\
& \geqq H_{\mu_{n}}\left(\mathscr{A}_{n} \mid \bigvee_{i=1}^{\infty} T_{n}^{-i} \mathscr{A}_{n}\right)-2 \varepsilon \\
& =h_{\mu_{n}}\left(T_{n}\right)-2 \varepsilon \\
& =e\left(T_{n}\right)-2 \varepsilon \geqq e(T)-2 \varepsilon .
\end{aligned}
$$

Since $\varepsilon>0$ is arbitrary we have $h_{\mu}(T) \geqq e(T)$ and the reverse inequality follows from (3.1). For a similar computation cf. [8], [10].

4. Linearisation of $(X, T)$. In this section we replace regional transitivity by the condition (1.2) and give sufficient conditions in order that there exists a normalised Borel measure $p$ on $X$ and a number $\beta \geqq 1$ such that $p T C=\beta p C$ for 
cylinders $C$ of $X$. We say that $T$ is open at $x$ if there exists a cylinder $\left(a_{0} \cdots a_{n}\right) \cap X$ of $X$ containing $x$ such that $T\left[\left(a_{0} \cdots a_{n}\right) \cap X\right]=\left(a_{1} \cdots a_{n}\right) \cap X$.

THEOREM 4. If $(X, T)$ is a symbolic dynamical system satisfying (1.2) such that $T$ is open at all but a countable number of points, and if in case e $(T)=0$ none of the exceptional points, where $T$ is not open, are periodic, then there exists a nonatomic normalised Borel measure $p$ which is positive on nonempty cylinders of $X$ and a number $\beta \geqq 1$ such that $p T C=\beta p C$ for all nonempty cylinders $C$ of $X$.

Proof. Let $\left(X_{n}, T_{n}\right)$ be the systems defined in the proof of Theorem 3, then $X_{n} \supset X_{n+1}$ and $\bigcap_{n} X_{n}=X$. Let $p_{n}, \beta_{n}$ be the measures and numbers defined by Theorem 2 such that

$$
p_{n} T_{n} C=\beta_{n} p_{n} C
$$

for cylinders $C$ of $X_{n}$. Let $\beta=\lim _{n \rightarrow \infty} \beta_{n} \geqq 1$ i.e. $e(T)=\log \beta$. Let $p$ be a limit point of the sequence $\left\{p_{n}\right\}$ in the dual space of the space of continuous functions on $X_{0}$. Again $p$ is concentrated on $X$. We show that $p T C=\beta p C$ for all cylinders $C$ of $X$. If $T$ is open at $x=a_{0} \cdots a_{k} \cdots a_{l} \cdots$ then there exists an integer $k$ such that $T\left[\left(a_{0} \cdots a_{l}\right) \cap X\right]=\left(a_{1} \cdots a_{l}\right) \cap X$ if $l \geqq k$. It follows also that $T\left[\left(a_{0} \cdots a_{l}\right) \cap X_{n}\right]=\left(a_{1} \cdots a_{l}\right) \cap X_{n}$ for all $n \geqq 0$. Choose $l \geqq k$, then

$$
\begin{aligned}
p T\left[\left(a_{0} \cdots a_{l}\right) \cap X\right] & =p\left(a_{1} \cdots a_{l}\right) \cap X=p\left(a_{1} \cdots a_{l}\right) \\
& =\lim p_{n}\left[\left(a_{1} \cdots a_{l}\right) \cap X_{n}\right]=\lim p_{n} T\left[\left(a_{0} \cdots a_{l}\right) \cap X_{n}\right] \\
& =\lim \beta_{n} p_{n}\left[\left(a_{0} \cdots a_{l}\right) \cap X_{n}\right]=\beta p\left[\left(a_{0} \cdots a_{l}\right) \cap X\right] .
\end{aligned}
$$

If the countable exceptional set where $T$ is not open has $p$ measure zero then the theorem is proved. If this set has positive measure then there exists a point $x$ such that $p\{x\}>0$. One can show by a similar technique to the above that for any point $x, p\{T x\} \geqq \beta p\{x\}$. Consequently $\beta=1$ and $e(T)=0$ if $p\{x\}>0$ for some $x$, and any such point $x$ is periodic. The hypothesis of the theorem implies, therefore, that no atoms exist among the exceptional points. Hence $p T C=\beta p C$ for all cylinders $C$ of $X$. From this it follows that no atoms whatsoever exist, for otherwise we would contradict (1.2). Finally, should $p(C)=0$ for some nonempty cylinder $C$ of $X$ we would have $p T^{n} C=0$ for $n=0,1, \cdots$ and $p(X) \leqq \sum_{n=0}^{\infty} p T^{n} C=0$.

5. Main theorem. Let $T^{\prime}$ be a strongly transitive piecewise monotone transformation of the unit interval $X^{\prime}$ onto itself. Let $A^{\prime}(i)$ be the open intervals $\left(a_{i}, a_{i+1}\right)$ on which $T^{\prime}$ is monotone and continuous.

Let $N$ be the smallest $T^{\prime}$ invariant set containing $S=\left\{a_{i}\right\} \cap X^{\prime}$. Then

$$
N=\bigcup_{m=0}^{\infty} T^{\prime-m} \bigcup_{n=0}^{\infty} T^{\prime n} S
$$

is countable. 
For each $x^{\prime} \in X^{\prime}-N$ define

$$
\phi\left(x^{\prime}\right)=x_{0}, x_{1}, \cdots
$$

where $T^{\prime n}\left(x^{\prime}\right) \in A^{\prime}\left(x_{n}\right), n=0,1, \cdots$. The set $\phi\left(X^{\prime}-N\right)$ of sequences of integers chosen from $Z=(0,1, \cdots, s)$ is a subset of

$$
X_{0}=\prod_{n=0}^{\infty} Z_{n} \text { where } Z_{n}=Z, \quad n=0,1, \cdots
$$

$\phi$ is a one-one map of $X^{\prime}-N$ into $X_{0}$. For otherwise, if $x^{\prime}<y^{\prime}$ and $\phi\left(x^{\prime}\right)=\phi\left(y^{\prime}\right)$ then for each $n, T^{\prime n}\left(x^{\prime}, y^{\prime}\right) \subset A^{\prime}\left(x_{n}\right)=A^{\prime}\left(y_{n}\right)$, since $T^{\prime}$ is monotone on each $A^{\prime}(i)$, and consequently $\bigcup_{n=0}^{\infty} T^{\prime n}\left(x^{\prime}, y^{\prime}\right) \subset \bigcup_{i=0}^{s} A^{\prime}(i) \neq X^{\prime}$, which contradicts the strong transitivity of $T^{\prime}$.

We consider $X_{0}$ as a topological space endowed with the compact metric totally disconnected product topology which arises from the discrete topology on $Z$. Let $X$ denote the closure of $\phi\left(X^{\prime}-N\right)$ in $X_{0}$.

We say that a finite sequence $b_{0}, b_{1}, \cdots, b_{n}$ is allowable if it begins some sequence in $\phi\left(X^{\prime}-N\right)$. It is not difficult to see that $X$ is the set of sequences $b_{0}, b_{1}, \cdots$ such that for every $n, b_{0}, \cdots, b_{n}$, is allowable. We denote by $T_{0}$ the shift transformation of $X_{0}$ onto itself. Evidently, $T_{0} \phi\left(X^{\prime}-N\right)=\phi\left(X^{\prime}-N\right), T_{0} X=X$ and $T_{0} \phi\left(x^{\prime}\right)$ $=\phi T(x)$ for $x^{\prime} \in X^{\prime}-N$. Let $T$ denote the restriction of $T_{0}$ to $X$.

LEMMA 1.

(i) $X-\phi\left(X^{\prime}-N\right)$ is countable.

(ii) For every nonempty cylinder $C$ of $X$ there exists an integer $m$ such that

$$
\bigcup_{n=0}^{m} T^{n} C=X
$$

(iii) $T$ is open at all points $x \in \phi\left(X^{\prime}-N\right)$ i.e. $T$ is open at all but a countable number of points.

Proof. (i) Suppose $x \in X-\phi\left(X^{\prime}-N\right), x=x_{0}, x_{1}, \cdots$ then for each integer $m$

$$
I_{m}=\bigcap_{n=0}^{m} T^{\prime-n} A^{\prime}\left(x_{n}\right) \neq \varnothing
$$

and either

$$
\bigcap_{n=0}^{\infty} T^{\prime-n} A^{\prime}\left(x_{n}\right)=\varnothing
$$

or it contains a single point in $N$. The latter can happen only a countable number of times. Let $M$ denote the set of sequences $x$ for which (5.1) and (5.2) hold. Each set $I_{m}$ is an open interval

$$
I_{m}=\left(a_{m}, b_{m}\right)
$$


Since (5.2) holds, there is a least integer $m_{0}$ such that either

$$
a_{m}=a_{m_{0}} \text { for } m \geqq m_{0}
$$

or)

$$
b_{m}=b_{m_{0}} \text { for } m \geqq m_{0} .
$$

We associate with $x$ the symbol $\left(x_{0}, x_{1}, \cdots, x_{m_{0}} ;+\right)$ if (5.3) holds and $\left(x_{0}, x_{1}, \cdots, x_{m_{0}} ;-\right)$ if (5.4) holds. This association is one-one and therefore $M$ is countable.

(ii) Let $C=\left(b_{0}, \cdots b_{n}\right) \cap X \neq \varnothing$ and $U=\bigcap_{k=0}^{n} T^{\prime-k} A^{\prime}\left(b_{k}\right)$. Since $T^{\prime}$ is strongly transitive, there exists an integer $m$ such that

$$
\bigcup_{k=0}^{m} T^{\prime k} U=X^{\prime}
$$

Therefore

$$
\bigcup_{k=0}^{m} T^{k} \phi(U-N)=\bigcup_{k=0}^{m} \phi T^{\prime k}(U-N)=\phi\left(X^{\prime}-N\right)
$$

i.e.

$$
\bigcup_{k=0}^{m} T^{k} C=\overline{\phi\left(X^{\prime}-N\right)}=X
$$

(iii) If $x \in \phi\left(X^{\prime}-N\right), x=x_{1} x_{2} \cdots=\phi\left(x^{\prime}\right), x^{\prime} \in X^{\prime}-N$ then we can choose $m$ so large that

$$
T^{\prime} A^{\prime}\left(x_{0}\right) \supset A^{\prime}\left(x_{1}\right) \cap T^{\prime-1} A^{\prime}\left(x_{1}\right) \cap \cdots \cap T^{\prime-m} A^{\prime}\left(x_{m}\right)
$$

since $T^{\prime} x^{\prime}$ is interior to the open set $T^{\prime} A^{\prime}\left(x_{0}\right)$.

Therefore

$$
T^{\prime}\left[\bigcap_{n=0}^{m} T^{\prime-n} A^{\prime}\left(x_{n}\right)\right]=\bigcap_{n=1}^{m} T^{\prime-n+1} A^{\prime}\left(x_{n}\right)
$$

and

$$
T^{\prime}\left[\bigcap_{n=0}^{m} T^{\prime-n}\left(A^{\prime}\left(x_{n}\right)-N\right)\right]=\bigcap_{n=1}^{m} T^{\prime-n+1}\left(A^{\prime}\left(x_{n}\right)-N\right) .
$$

If we apply $\phi$ to each side of (5.5) we get

$$
T\left[\left(x_{0} \cdots x_{m}\right) \cap \phi\left(X^{\prime}-N\right)\right]=\left(x_{1} \cdots x_{m}\right) \cap \phi\left(X^{\prime}-N\right)
$$

and

$$
T\left[\left(x_{0} \cdots x_{m}\right) \cap X\right]=\left(x_{1} \cdots x_{m}\right) \cap X
$$

i.e., $T$ is open at each $x \in \phi\left(X^{\prime}-N\right)$. 
THEOREM 5. Let $T^{\prime}$ be a strongly transitive piecewise monotone transformation of $X^{\prime}$ onto itself.

If either

(i) $T^{\prime} A^{\prime}(i) \cap T^{\prime} A^{\prime}(j) \neq \varnothing$ for some $i \neq j$ or

(ii) $T^{\prime} A^{\prime}(i) \cap T^{\prime} A^{\prime}(j)=\varnothing$ for all $i, j=0,1, \cdots, s \quad(i \neq j)$ and $T^{\prime}$ has no periodic points then $T^{\prime}$ is conjugate to a uniformly piecewise linear transformation.

Proof. If (i) is satisfied then $T^{\prime}$ has at least two inverse images for each point of an open interval. Since a finite number $m$ of iterates of this interval cover $X^{\prime}, T^{\prime m}$ has at least two inverse images for all points of $X^{\prime}$. As $T^{m}$ will have the same property it follows easily that

$$
m e(T)=e\left(T^{m}\right) \geqq \log 2 .
$$

Consequently, either $e(T)>0$ or (ii) is satisfied.

Therefore the conditions of Theorem 4 are satisfied. Let $p$ be the measure defined by Theorem 4. Since $p$ is nonatomic and $X-\phi\left(X^{\prime}-N\right)$ is countable we can regard $p$ as a measure on $\phi\left(X^{\prime}-N\right)$. Let $p^{\prime}$ be the measure defined on Borel subsets of $X^{\prime}$ by $p^{\prime}(E)=p \phi(E-N)$, then $p^{\prime}$ is a nonatomic measure which is positive on nonempty open subsets of $X^{\prime}$ and

$$
p^{\prime} T^{\prime}(E)=\beta p^{\prime}(E)
$$

for each Borel subset $E$ of $A^{\prime}(i), i=0, \cdots, s$.

Let $\psi\left(x^{\prime}\right)=p^{\prime}\left[0, x^{\prime}\right], x^{\prime} \in X^{\prime}$ then $\psi$ is a homeomorphism of $X^{\prime}$ onto itself and

$$
l \psi(E)=p^{\prime}(E)
$$

where $l$ is Lebesgue measure.

Let $S^{\prime}\left(x^{\prime}\right)=\psi T^{\prime} \psi^{-1}\left(x^{\prime}\right)$ then

$$
\begin{aligned}
l S^{\prime}(E) & =l \psi T^{\prime} \psi^{-1}(E)=p^{\prime} T^{\prime} \psi^{-1}(E) \\
& =\beta p^{\prime} \psi^{-1}(E) \quad\left(\text { if } \psi^{-1}(E) \subset A^{\prime}(i)\right) \\
& =\beta l(E) \quad\left(\text { if } E \subset \psi A^{\prime}(i)\right) .
\end{aligned}
$$

Since $S^{\prime}$ is homeomorphic with $T^{\prime}, S^{\prime}$ is a piecewise monotone transformation and on each interval $\psi A^{\prime}(i), S^{\prime}$ is linear by (5.6). Therefore, there exist $\alpha_{0}, \cdots, \alpha$ such that

$$
S^{\prime}\left(x^{\prime}\right)=\alpha_{i} \pm \beta x^{\prime}, \quad x^{\prime} \in \psi A^{\prime}(i)
$$

i.e.,

$S^{\prime}$ is piecewise linear. 


\section{Applications.}

Corollary 1 (PoInCARÉ-DenJoy). If $T^{\prime}$ is a homeomorphism of the circle $X^{\prime}$ onto itself such that for each open set

$$
\bigcup_{n=0}^{\infty} T^{\prime n} U=X^{\prime}
$$

then $T^{\prime}$ is conjugate to a translation of $X^{\prime}$.

Proof. The circle is homeomorphic to the unit interval $[0,1)$ with end points identified. The compactness of the circle and the absence of periodic points (which follows from (6.1)), implies the conditions of Theorem 5 .

Let $X^{\prime}=[0,1)$ and let $f$ be a strictly increasing continuous function with domain $[0, t)(t>1)$ and range $X^{\prime}$ and let

$$
T^{\prime}\left(x^{\prime}\right)=\left(f^{-1} x\right)
$$

where $(y)=y-[y]$ is the fractional part of $y$. These transformations were discussed by Rényi [5], and the uniformly piecewise linear transformations $S^{\prime}$ of this type arise from functions $f$ of the form

$$
f\left(x^{\prime}\right)=\frac{x^{\prime}}{\beta}, \quad \beta>1 \quad\left(0 \leqq x^{\prime}<\beta\right)
$$

$$
S^{\prime}\left(x^{\prime}\right)=\left(\beta x^{\prime}\right)
$$

COROLlaRY 2. If $T^{\prime}$ is a strongly transitive transformation of type (6.2) (for example if $f$ is differentiable and $\left.0<f^{\prime}(x)<1,[5]\right)$ then $T^{\prime}$ is conjugate to a transformation of type (6.3).

Proof. Condition (i) of Theorem 5 is satisfied. Rényi has proved the existence of a normalised measure equivalent to Lebesgue measure which is preserved by the transformation (6.3). In [11] and [12] its explicit form is given. It follows, therefore, that strongly transitive transformations of type (6.2) preserve a continuous measure which is positive on open sets.

COROLlaRY 3. If $T^{\prime}$ is a strongly transitive continuous transformation of the unit interval (with or without each end point) with only a finite number of maxima, and minima, then $T$ is conjugate to a continuous uniformly piecewise linear transformation.

Proof. For $T^{\prime}$ to be strongly transitive it is necessary that there be at least one turning point. Consequently, condition (i) of Theorem 5 is satisfied.

This corollary answers, partially, a question of Ulam's [3], who states that it might be of importance in the study of iterations of functions of a real variable. 


\section{WILLIAM PARRY}

\section{REFERENCES}

1. W. Parry, Intrinsic Markov chains, Trans. Amer. Math. Soc. 112 (1964), 55-66.

2. E. R. Van Kampen, The topological transformations of a simple closed curve onto itself, Amer. J. Math. 57 (1935), 142-152.

3. S. M. Ulam, Problems in modern mathematics, Wiley, New York, 1964.

4. P. R. Stein and S. M. Ulam, Non-linear transformation studies on electronic computers, Rozprawy Mat. 39 (1964).

5. A. Rényi, Representations for real numbers and their ergodic properties, Acta. Math. Acad. Sci. Hungar. 8 (1957), 477-493.

6. W. H. Gottschalk and G. A. Hedlund, Topological dynamics, Amer. Math. Soc. Coll. of Publ. Vol. 36, Amer. Math. Soc., Providence, R. I., 1955.

7. R. L. Adler, A. G. Konheim and M. H. McAndrew, Topological entropy, Trans. Amcr. Math. Soc. 114 (1965), 309-319.

8. K. Jacobs, Ergodic decomposition of the Kolmogoroff-Sinai invariant, Proc. Internat. Sympos. Ergodic Theory, Academic Press, New York, 1963, pp. 173-190.

9. A. I. Khinchin, Mathematical foundations of information theory, Dover, New York, 1957.

10. L. Breiman, On achieving channel capacity in finite memory channels, Illinois J. Math. 4 (1960), 246-252.

11. A. O. Gelfond, On a general property of number systems, Izv. Akad. Nauk SSSR 23 (1959), 809-814. (Russian)

12. W. Parry, On the $\beta$-expansions of real numbers, Acta. Math. Acad. Sci. Hungar. 11 (1960), 401-416.

UNIVERSITY OF SUSSEX,

FALmer, Brighton, ENGland 